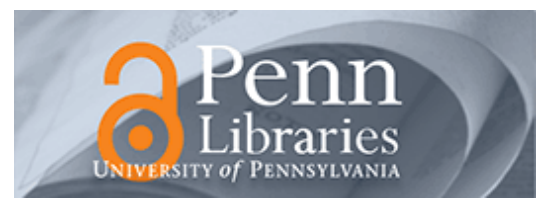

University of Pennsylvania

ScholarlyCommons

Marketing Papers

Wharton Faculty Research

$10-2007$

\title{
Biases in Predicting Preferences for the Whole Visual Patterns from Product Fragments
}

Shenghui Zhao

Robert J. Meyer

University of Pennsylvania

Follow this and additional works at: https://repository.upenn.edu/marketing_papers

Part of the Advertising and Promotion Management Commons, Behavioral Economics Commons, Business Intelligence Commons, Cognition and Perception Commons, Experimental Analysis of Behavior Commons, Marketing Commons, and the Sales and Merchandising Commons

\section{Recommended Citation}

Zhao, S., \& Meyer, R. J. (2007). Biases in Predicting Preferences for the Whole Visual Patterns from Product Fragments. Journal of Consumer Psychology, 17 (4), 292-304. http://dx.doi.org/10.1016/ S1057-7408(07)70039-6

This paper is posted at ScholarlyCommons. https://repository.upenn.edu/marketing_papers/328

For more information, please contact repository@pobox.upenn.edu. 


\title{
Biases in Predicting Preferences for the Whole Visual Patterns from Product Fragments
}

\begin{abstract}
This research examines the ability of consumers to predict the appeal of complete visual patterns from small sample fragments. In a task designed to mimic the dilemma of choosing wallpaper from small swatches, study participants are shown fragments taken from a large pattern design and are asked to predict how attractive they would find the complete image. Drawing on prior research on affective forecasting, predictions are hypothesized to be driven by an anchoring-and-adjustment process that skews forecasts toward the attractiveness of fragments when judged in isolation. Results from 3 laboratory studies support this basic hypothesis: Respondents consistently overestimate the degree to which their initial reactions to fragments predict their subsequent evaluations of wholes. The size of this projection bias is, in turn, conditioned by such moderators as prior familiarity with product fragment, cognitive load, and visualization abilities-effects that are consistent with an anchoring-and-adjustment explanation for the data.
\end{abstract}

\section{Disciplines}

Advertising and Promotion Management | Behavioral Economics | Business | Business Intelligence | Cognition and Perception | Experimental Analysis of Behavior | Marketing | Sales and Merchandising 
Biases in Predicting Preferences for the Whole from Product Fragments 
Biases in Predicting Preferences for the Whole from Product Fragments

\begin{abstract}
In this research we examine the ability of consumers to predict the likely appeal of large visual patterns from small sample fragments. In a task designed to mimic the dilemma of choosing wall paper from small swatches, study participants are shown fragments taken from a large pattern design and are asked to predict how attractive they would find the complete image. Drawing on research on affective forecasting and inference heuristics, we hypothesize that these predictions will be driven by an anchoring-and-adjustment process that skews predictions toward the local attractiveness of fragments. Results from three laboratory studies support this basic hypothesis: respondents consistently overestimate the degree to which their initial reactions to fragments predict their subsequent evaluations of wholes. The size of this projection bias is, in turn, conditioned by such moderators as prior familiarity with product fragment, cognitive load, and visualization abilities — effects that are consistent with an anchoring-and-adjustment explanation for the data.
\end{abstract}


Many purchase decisions require consumers to imagine whole products from fragmentary images. When homeowners decide what color to paint a room they often have little more to go on than small color swatches provided by a retailer, fragments from which whole rooms must be imagined. Likewise, shoppers from apparel catalogs such as Land's End face a similar dilemma of visualization: not only must they imagine what a garment and pattern would look viewed at full scale, but, like paint shoppers, what a depicted garment might look like given a different color or pattern option. Finally, even moviegoers cannot avoid the need to mentally extrapolate: decisions about whether to attend a movie are often made based on snippets of content contained in trailers or print ads.

How skilled are consumers at predicting their reactions to product wholes from small fragments? While we are aware of no research that has examined this question explicitly, the rapid growth of computer-based consumer visualization aids suggests that, at the very least, it is not a task that consumers naturally perform easily or well. The Dutch Boy web site, for example, features a visualization tool that allows consumers to see what different paint color and trim combinations might look like when applied to a prototypical room; and Toyota's Scion site allows consumers to assemble and view virtual cars with different combinations of body accessories and colors. Yet, despite the prevalence of such purchase decisions, surprisingly little is known about how mental extrapolation problems are solved in the absence of aids, or the kinds of biases with which they are associated.

The purpose of this research is to propose and test a process model of consumer judgment in a class of mental extrapolation tasks where consumers view a small swatch taken from a much larger overall pattern (such as a wall paper or a garment) and are asked to predict their liking for the final composite. After a time delay they are shown the overall composite pattern and evaluate 
the composite. Drawing from the recent literatures on affective forecasting and inference heuristics, we hypothesize that these mental extrapolation judgments will be driven by an anchoring-and-adjustment process that skews affective forecasts toward the initial aesthetic appeal of product fragments. Hence, consumers will tend to overestimate how much they will like large images created from small attractive fragments and dislike large images created from unattractive fragments. Three laboratory studies support this basic idea, as well as find evidence for the effect of several moderators that follow from anchoring theory. Specifically, the discrepancy between predicted likings and evaluations of the final product is found to be more pronounced when consumers have prior exposure to the fragments in different contexts or experience cognitive load while making predictions, and are mollified when consumers have strong visualization skills.

The paper is organized in three sections. We first review the relevant background literatures and develop several hypotheses about the judgmental processes and accuracy in such extrapolation judgments. We then reports three laboratory studies testing these hypotheses, and conclude with a discussion of the implications of the work and suggestions for future research.

\section{Hedonic Prediction from Product Visualizations}

Consider the following common dilemma. A consumer has made the decision to apply a new wall paper to a room, and travels to the local home store to see what alternative new patterns might be available. While she is pleased to see the wide assortment, she observes that they are viewable only as small swatches in catalogs. Her decision will thus not be an easy one; beyond the difficulty posed by the breadth of options, the key criterion on which the choice should be made - how a wall paper will look once fully installed in a room at home - is not something she can observe. The decision will thus test her ability to accurately imagine the room at home with 
different new wall papers and anticipate how she would affectively respond to these different options.

How might she solve this mental extrapolation problem? Although there has been extensive research on the cognitive processing of mental images in both psychology and marketing, little of the work is directly related to the mental extrapolation problem considered here. The work on mental images in psychology has primarily dealt with the speed of generating and processing mental images (e.g., rotating the letter "R"; see, e.g., Kosslyn, 1994; Shepard \& Cooper, 1982), and is not concerned with the evaluative consequences of such operations. Likewise, research on imagery in marketing is primarily interested in comparing cognitive processing of mental imagery and verbal information in product evaluations and new product design (see Dahl, Chattopadhyay, \& Gorn, 1999; Keller \& Block, 1997; MacInnis \& Price, 1987). As such, its relevance is again limited. It does not, for example, comment on the specific processes of visualization or its accuracy. Finally, the literature on product judgment given limited attribute information (e.g., Kardes, Posavac, \& Cronley, 2004; Meyer, 1981; Simmons \& Lynch, 1991) examines how product evaluations are influenced by missing attribute values. While this literature shares with the current research a common interest in product judgments under uncertainty, the focus in that work is more on how consumers form inferences about the value of specific attributes (e.g., unknown quality given a known price) rather than holistic visualization of products from fragmentary descriptions.

In contrast, a body of work that potentially has strong relevance to the present research is the recent literature on affective forecasting—-research that explores people's ability to predict their future affective response to a focal event such as a job change (for reviews, see Loewenstein \& Schkade,1999; Wilson \& Gilbert, 2003). The common finding is that when 
making such forecasts people often overestimate how long their immediate affective reactions to an event will persist in the future. Hence, academics facing the prospect of being denied tenure underestimate their ability to rebound from disappointment, and hungry consumers find the prospect of eating dinner items for breakfast more attractive than satiated people (Gilbert, Gill, \& Wilson, 2002; Wilson, Wheatley, Meyers, Gilbert, \& Axsom, 2000). A widely accepted explanation is that people make forecasts using an anchoring-and-adjustment strategy that puts too much weight on current emotional or affective states (e.g., Kahneman \& Schkade, 1999; Loewenstein, O’Donoghue, \& Rabin, 2003; Wilson et al., 2000). Under anchoring theory projection biases are thus most acute when the event that triggers these emotions is particularly salient, or the task of envisioning the future is particularly difficult. As an example, Gilbert et al. (2002) found that the tendency for hungry people to have a strong preference for consuming dinner items for breakfast is exacerbated when respondents make the forecast while having to perform other cognitive tasks.

Although the evidence for such anchoring biases has been drawn from contexts that are quite different from the mental visualization context considered here, the tasks nevertheless hold an obvious parallel: both involve prospective judgments about the value of an unseen larger epoch (in our case, a whole visual image) based on small seen component (here, a swatch or fragment). The major difference, of course, is that ours is a task of spatial rather than temporal extrapolation; we examine people's ability to look beyond their immediate reactions to an image fragment to envision how they would react to a larger, more complete, image.

\section{Visualizations as Affective Forecasts}

How might consumers undertake visualizations? Intuition gives rise to two major process hypotheses. The most straightforward is holistic mental imaging: upon viewing a fragment the 
consumer would construct an explicit mental image of what the larger pattern would look like (perhaps drawing from examples in memory), much as one might try to mentally picture how a room at home might look adorned in a certain color while at a paint store. The consumer's judgment would then be the attractiveness of this holistic image as it appears in his or her mind's eye. Note that this mental construction need not be effortful: while in some cases it might indeed correspond to the stereotypic image of a consumer closing his or her eyes to picture a room painted in a certain color, in other cases the fragment might automatically trigger recall of a familiar completed image (such as a room painted in similar colors that one has seen before). In either case the predicted affect is that associated with this envisioned whole image.

But what if a fragment does not give rise to such a specific visualization (as might be the case for an unfamiliar abstract shape), or the consumer lacks good visualization skills? In such cases we hypothesize that the projection task would be solved using a numeric adjustment heuristic not unlike that used to make hedonic forecasts in other domains. That is, the consumer would use the attractiveness of the fragment in isolation as a starting point for the forecast and then adjust this valuation up or down using heuristic knowledge about how the appeal of complete patterns typically tends to differ from that of a fragment. For example, if a consumer is attracted to a wall paper swatch because it contains a finely-detailed image of a flower, he or she might conjecture that this appeal would be lost in a wall paper that contained many of such flowers, where the details and uniqueness of the fragment image would be difficult to discern.

\section{Fragments as Anchors in Holistic Forecasts}

Central to this research is a hypothesis that regardless of which of these processes consumers use to make forecasts—or whether they use a mixture — predicted evaluations of whole images from fragments will exhibit an anchoring bias similar to that which marks 
affective forecasts in temporal prediction tasks. That is, initial exposure to fragments that trigger positive associations will lead to forecasts of the attractiveness of wholes that are too positive, while initial exposure to fragments that trigger negative associations will lead to forecasts that are too negative.

The rationale for this hypothesis is twofold. When a consumer first sees a fragmentary image containing recognizable elements (such as a swatch from a flowered pattern of wall paper) these elements will often trigger an affective response that is independent of later-formed beliefs about the completed image. An example might be the spontaneous feeling of pleasure one might feel upon first seeing a favorite color or image (e.g., Zajonc, 1980). Even if the consumer later concludes that a completed image could look quite different from this fragment, prior work on anchoring suggests that this initial disposition will nevertheless have a sustained effect on the subsequent holistic judgment (e.g., Forster, 2004; Tverksy \& Kahneman, 1974). In our case, this could occur mechanically either by making it more likely to retrieve from memory completed mental images that are affectively congruent with the prior disposition (e.g., envision only attractive rooms after being primed by an attractive color swatch) or provide numerical estimates of the attractiveness of wholes that are congruent with the prior (e.g., Chapman \& Johnson, 1999).

This effect would be exacerbated in the current context by comparative visual salience of fragments. The immediately-felt affect for a tangible fragment will tend to loom psychologically large compared to the imagined affect for an imagined whole, giving it more weight in any composite judgment (e.g., Wilson et al., 2000). Put another way, even if consumers imagine that their affective response to a complete image would be more tempered than that which they are 
currently feeling about the fragment, it will be hard for them to prevent their prospective judgments from being colored by these immediate feelings.

It is important to emphasize that the empirical validity of this hypothesis hinges on consumers having an independent affective response to fragments when they are first viewedresponses that condition their subsequent forecast of the attractiveness of completed patterns. The possibility exists, of course, that no such pre-processing occurs; exposure to a fragment may immediately trigger a mental image of a completed pattern which becomes the focus of the projected valuation. Under such a process predictive accuracy would entirely depend on the empirical correspondence between the imagined image and the realized one, a correspondence that might just as easily be overly positive as overly negative. Hence, in principle, the finding of an anchoring effect provides joint validation of the existence of a staged constructive process as well as the biasing effect of this process.

The problem with such a conclusion, however, is that judgment data that look like anchoring effects could arise simply as a statistical artifact of regression-to-the-mean effects in repeated testing, even if consumers' forecasts are unbiased. If forecasts and subsequent valuations are measured with error, subsamples of consumers who show the highest and lowest mean predicted affect ratings at time 1 will naturally tend to have more moderate mean ratings at time 2 - an effect that could be confused with real over- and under-prediction of affect. A more thorough analysis of the process that generates predicted valuations of wholes, therefore, requires data showing that the strength of the anchoring effect-if it exists—-is conditioned by a set of moderators predicted by anchoring theory. These moderators will be explored in turn. 


\section{Moderators of Visualization Accuracy}

Fragment Familiarity. An emerging theory of the anchoring bias in recent years views the anchoring effect as the product of biased activation and retrieval in memory processes (e.g., Chapman \& Johnson, 1999; Mussweiler \& Strack, 1999). According to this anchoring-asactivation account, the decision maker uses the anchor as a memory probe which, in turn, activates information that is consistent with the anchor more than the information that is inconsistent. In support of this account, increased elaboration of anchors leads to a stronger anchoring effect (Wilson et al., 1996) while directing decision maker's attention to anchorinconsistent information substantially reduces the bias (Chapman \& Johnson, 1999).

A natural hypothesis that follows from such work is that if consumers make forecasts of the value of holistic images using a staged constructive process, we should observe a negative correlation between fragment familiarity and the accuracy of predictions of the attractiveness of completed whole images. Specifically, because more familiar fragments are likely to be the subject of greater cognitive elaboration (e.g., a fragment that contains images of a familiar location may prime memories of an enjoyable vacation), the more these cognitions will interfere with competing attempts to form unbiased mental images of what a completed whole images might look like. As such, affective reactions to the fragment will act as a stronger anchor on predicted valuations of the whole.

The obvious caveat to this prediction, of course, is that such a relationship would not hold if the overall pattern is as familiar as the fragment itself-as might be the case for certain familiar prints that are uniquely recognizable from small swatches. Rather, our hypothesis centers on those cases where the consumer has never seen the holistic pattern made from the fragment. 
Cognitive load. A closely related moderator is cognitive load. One of the major empirical findings of previous work on anchoring effects is that anchoring effects are exacerbated in tasks that impose a constraints on available cognitive capacity—such as making decision makers memorize numbers while formulating inferences (e.g., Gilbert et al., 2002). We thus might expect a similar finding here: because the task of mentally visualizing a completed image (and constructing a predicted valuation) requires cognitive resources, the greater the cognitive load that is placed on consumers when making a projective judgment, the worse their predictions should be on average, and the more they will tend to be anchored by the independent attractiveness of the fragment. To illustrate, we suggest that it is this moderator that makes the task of picking fabrics from large books of swatches so daunting for consumers; when trying to make choices consumers may be able to do little more than react to the immediate attractiveness of the swatches, an impression that might be quite different from that which would be formed if considerably more time was spent considering what each swatch would look like on a larger scale.

Visualization Skills. The ability to visualize patterns obviously varies from person to person. While the average consumers are often helplessly confused in front of a wall of paint colors in Home Depot, professional interior designers have the knack to arrange furniture in their "mind's eye" for maximal aesthetic appeal. The stark contrast between professional interior designers and the average consumers in their ability to visualize patterns and objects probably reflect the difference in experience and training. Even with ordinary consumers, people often have different preferences between visual and verbal stimuli and hence their ability to process each type of stimuli also differs. Visualizers and verbalizers differ in their propensity to process visual materials and to generate and rely on mental imagery in their decision making, and they 
differ in their ability to process visual stimuli. If professional interior designers are a guide, we should expect those consumers who are more adept at processing visual stimuli to perform better at the visualization task and produce smaller errors than those who prefer verbal stimuli.

Experience with visualization tasks. Finally, experience with visualization tasks is another factor that may have beneficial effect on forecast accuracy. A few precedents for a beneficial effect of task experience have been reported in the affective forecasting literature. Brickman, Coates, and Janoff-Bulman (1978), for example, have noted that although most people tend to ignore the impact of hedonic adaptation when predicting future feelings, those who have witnessed their friends or relatives adapting to the life of a paraplegic are more aware of adaptation effect in their judgments. Similarly, Ubel, Loewenstein, and Jepson (2005) effectively reduced the anchoring bias in their participants by priming them with examples of hedonic adaptation. We thus might expect a similar experience effect here: a consumer who repeatedly finds themselves being disappointed with the attractiveness of completed images (e.g., how a room actually looks when painted in dark blue) might well develop skills that enhance predictive accuracy such as by learning to ignore the initial affective signals provided by the fragment. Indeed, anecdotal evidence supporting such training effects is implied by the existence of design consultants, people whose ability to accurately anticipate the attractiveness of wholes from parts stems not just from innate visualization skills (as discussed above), but also sheer experience gained through trial and error.

\section{Overview of the Studies}

Three laboratory studies are reported below that explore the accuracy and processes of extrapolation judgments from product fragments. All three studies followed the same basic procedure: participants first viewed fragments taken from larger whole images, and made 
predictions about how attractive they would find the whole. After a delay they were shown the completed image, and provide a rating of its holistic attractiveness. Our central interest was the discrepancy between predicted liking based on fragments and actual evaluations of the wholes. Study 1 tested the basic anchoring hypothesis while studies 2 and 3 explored the influence of potential moderators.

Study 1

\section{Method}

Participants. Forty-six business undergraduate students at a large east coast university participated in the study as part of an experimental session that included other unrelated experiments. Each participant was paid $\$ 10$ for their participation. Two participants did not respond to all the questions and were thus eliminated from analysis reported below, leading to an effective sample size of forty-four.

Stimuli. Each participant examined a total of eighteen fragments with varying level of attractiveness. The fragments were selected from a large sample of patterns drawn from several online sources such as Microsoft's Clip Art. Figure 1 (lower panel) provides some sample fragments. Since pretests with student volunteers from the same university showed a high level of heterogeneity regarding the perceived attractiveness of those fragments, we decided to measure, rather than manipulate, fragment attractiveness within-subjects.

Insert Figure 1 about here

Procedure. The study consisted of three phases, each separated from the others by a 5-to10-minute filler task. In phase 1, participants were told that they just moved in a new apartment 
and needed to get a new wall paper for their living room. Then they were shown a series of fragment patterns that they were told were samples of wall papers available at a local home improvement store. After each fragment was shown on the computer screen, they were asked to rate the fragment on a 15-point scale ( $1=$ "Very Ugly" and $15=$ "Very Attractive"). In phase 2, the fragment was shown side by side with a sample wall paper (i.e., referent) that depicted how the fragment pattern would be duplicated to form the completed design (e.g., a square grid; see Figure 1, upper panel). Participants were then asked to predict how much they would like the wall paper with that fragment in their living room. These predictions were made on the same 15point scale as in phase 1 . To minimize boredom, several sample wall papers were used and their pairing with the target fragments was counterbalanced. In phase 3 of the study, participants were shown wall papers on the computer screen which were constructed with the fragments they viewed earlier in phase 1. Each wall paper occupied the entire computer screen. Participants were asked to indicate their liking of each wall paper on the 15-point scale used in phase 1 of the study. All experimental materials were delivered on computer screens, and participants completed the study at their own pace.

In addition to evaluative judgments, the computer program also measured the response time for each prediction. Our goal in measuring response times was to explore whether initial hedonic reactions to the fragment would influence not just the predicted liking of the whole but also the evaluation (or visualization) process itself. For example, prior research on response latencies has shown that negative stimuli often trigger faster, more automated, response processes (e.g., Fiske, 1980; Gehring \& Willoughby, 2002). As such, we may expect shorter response times for unattractive fragments than for attractive ones. 
Results

Preliminaries. Mean evaluations of the fragments, predicted liking of wall papers based on fragments (henceforward predicted liking), and evaluation of the wall paper (henceforward experienced liking) can be found in Table 1. The mean rating of fragments was close to neutral $(M=7.03)$ with substantial variation both within and between participants. Their predicted liking of wall papers based on fragments was, on average, lower than their evaluation of the corresponding fragment $(M=6.34), F(1,43)=44.41, p<.0001$, as was experienced liking $(M=$ 6.16), $F(1,43)=20.57, p<.0001$.

Insert Table 1 about here

Influence of Fragment Attractiveness. We hypothesized that predicted likings would be more strongly influenced by fragment evaluations than would experienced likings. Predicted and experienced likings were separately regressed on fragment evaluations, after controlling for participant specific effects. The regression results confirmed this conjecture. Specifically, fragment evaluations had a strong effect on predicted liking, $\beta=.86, t(747)=44.81, R^{2}=.79$. In contrast, the effect of fragment evaluations on experienced likings, though significant, was much more modest, $\beta=.46, t(747)=14.48, R^{2}=.41$. The difference in $\beta$ 's was highly significant, repeated measures $F(1,747)=140.92, p<.0001$. Not surprisingly, fragment evaluations had a stronger correlation with predicted likings $(r=.85)$ than with experienced likings $(r=.47)$, paired $t(43)=9.76, p<.0001$, a pattern that was observed for all but two (or, 95\%) participants.

Congruence between Prediction and Evaluation of Whole Product. Next we examine the consistency between predicted and experienced likings. For this purpose, we calculated the 
discrepancy as predicted liking - experienced liking. At the aggregate level, predicted liking was not different from experienced liking ( $M=.18$ for discrepancy), $t(791)=1.39, p>.15$. However, as predicted by the anchoring hypothesis, the discrepancies showed a significant positive relationship to fragment evaluation, $\beta=.40, t(747)=11.87, p<.0001, R^{2}=.27$. In other words, participants were more likely to overestimate their liking for wall papers constructed from fragments that they found appealing, but more likely to underestimate their liking for those from fragments that were found to be unappealing.

To further illustrate the effect, fragments were median split into attractive or unattractive as a within-subjects factor based on each participant's own ratings. Discrepancies between predictions and evaluations of whole products were subjected to a one-way repeated measures ANOVA with fragment attractiveness as the independent variable. The mean discrepancy was negative for unattractive fragments $(M=-.91)$ but positive for attractive fragments $(M=1.20)$, $F(1,704)=84.45, p<.0001$. That is, compared to actual evaluations of the wholes, predictions were overly optimistic for attractive fragments but overly negative for unattractive ones.

Response Time. Response time data for predicted likings were first log-transformed because of non-normality and then submitted to a one-way repeated measures ANOVA with fragment attractiveness as the independent variable. Consistent with prior work on the processing of negative versus positive cues (e.g., Fiske, 1980), response times were significantly longer for attractive fragments than for unattractive ones ( $M=4.7$ vs. 4.1 seconds), $F(1,704)=6.29, p<$ .05 .

\section{Discussion}

Study 1 provided basic support for the hypothesis that visual extrapolation judgments would be prone to a fragment-induced anchoring bias that parallels the projection biases found in 
other domains of hedonic forecasting. As predicted, predictions about the overall attractiveness of wholes were overly influenced by the initial independent attractiveness of fragments, such that forecasts in response to attractive fragments proved overly optimistic and those for unattractive fragments proved overly pessimistic. The data also produced tentative evidence that the initial attractiveness of fragments also influenced the process that produced the predictions.

Specifically, positive fragments were associated with longer response latencies, suggesting that participants engaged in greater mental elaboration after viewing attractive (vs. unattractive) fragments.

Two concerns, however, limit the strength of the conclusions that can be drawn from the study. One is that the task faced by participants differed in several ways from that which characterizes many real-world extrapolation tasks such as choosing a real wall paper at a home store. While consumers will almost always have a referent context in mind when evaluating a fragment (e.g., their living room), it will not always be seen side-by-side with the fragment, hence require more active imagination (e.g., imaging how a couch or room would look like, not just a larger section of wall paper). More active visualization might, in turn, offset the anchoring effects observed here. In addition, the finding of a statistical carry-over effect between fragment evaluations and forecasts provides only suggestive evidence for an anchoring-and-adjustment process. Stronger evidence requires us to show not only that the results of the first study replicate in a more realistic context, but are also conditioned by moderators theorized by anchoring theory. In Study 2 we undertake the first of two such analyses. 
The primary goals of study 2 were twofold. First, we sought to replicate the key results of study 1 in a more realistic task setting. Second, we sought to examine the process of extrapolation judgments more closely by studying the effect of fragment familiarity on the congruence between fragment-based predictions and evaluations of wholes. Based on the activation account of the anchoring bias, we hypothesized that prior familiarity or exposures to the fragment would reduce the congruence between predictions and whole product evaluations. We also used the study to remedy a possible methodological weakness of Study 1, our use of the same evaluation scale for both fragment evaluation and prediction. The concern is that the use of the same numeric scale may have artificially inflated the observed anchoring effect by enhancing recall of fragment evaluations at the time that holistic forecasts were made-a source of fragment salience that would not occur in natural settings.

\section{Method}

Eighty-six students from the same university as in study 1 participated in the study for partial course credit. Participants were told to imagine that they were buying a shirt from a website, and were shown a series of swatches of the fabrics that would be used to make the shirt. In order to more closely mimic the kinds of constraints on visualization that often arise in purchase contexts, unlike in Study 1 participants were not provided with a detailed illustration of exactly how the pattern would be arrayed in the final shirt. They were simply shown a generic illustration of the kind of shirt they would be buying (an illustration of a white short-sleeved shirt) and told that the patterns would be arrayed in a grid layout.

The procedure closely followed that of study 1, so we only note the differences here. In phase 1, participants evaluated a series of pattern swatches on both their prior familiarity with each swatch and its attractiveness. Prior familiarity was measured on a binary scale (the question 
asks, "Does the following fragment appear familiar to you?" with "No" and "Yes" as response options). Given the concern over the use of the same scales for fragment evaluations and predictions, the attractiveness was measured on a 7-point bipolar scale $(-3=$ "Very Ugly" and +3 = "Very Attractive"; fragments were categorized as attractive if their ratings were positive and unattractive if they were negative.). In contrast, participants made predictions in phase 2 and evaluated the whole shirts in phase 3 on a 101-point scale $(0=$ "Very Ugly" and $100=$ "Very Attractive"). Figure 2 provides a screenshot for the prediction task as well as some sample stimuli.

Insert Figure 2 about here

Results

Table 2 provides the means on the following measures for each type of fragments: 1 ) attractiveness rating for fragments, 2) predicted liking from fragments, 3) evaluation of the whole shirt, and 4) discrepancy for each prediction (= predicted liking from fragments evaluation of the whole shirt).

Insert Table 2 about here

Influence of Fragment Attractiveness. Mirroring the findings in study 1, fragment evaluations had a strong influence on predicted liking, $\beta=5.11, t(4427)=13.46, p<.0001, R^{2}=$ .39. The effect of fragment evaluations was significantly stronger for attractive fragments than for unattractive ones, $F(1,4426)=11.20, p<.001$. The same anchoring effect was also observed 
for evaluations of the whole, though as in study 1 , the effect was weaker than for predicted likings, $\beta=4.21, t(4427)=9.07, p<.0001, R^{2}=.36$. The difference in $\beta$ 's was statistically significant, $F(1,8840)=2.95, p<.05$. Consistent with these findings, fragment evaluations had higher correlations with predicted likings $(r=.51)$ than with experienced likings $(r=.37)$, paired $t(83)=6.39, p<.01$. This pattern of results was evident among $78 \%$ of the participants.

In addition, this anchoring effect was also evident in discrepancy between predictions and actual likings of the whole products, $\beta=.84, t(4426)=4.28, p<.05, R^{2}=.10$. Consistent with the results of study 1 , the data indicated that the discrepancy was more pronounced for attractive than for unattractive fragments $(M=8.92$ vs. 3.29$), F(1,84)=17.25, p<.0001$. However, unlike study 1 , predicted likings generally tended to exceed evaluations of whole products, even when fragments were considered unappealing (see Table 2). This was due to the surprisingly low evaluations of the completed shirts.

Effects of Fragment Familiarity. We hypothesized that fragment familiarity would moderate the anchoring effect in predicted likings. Consistent with this prediction, the data revealed a significant fragment familiarity $\mathrm{x}$ fragment attractiveness interaction on predicted liking, $F(1,4426)=6.35, p<.01$. Specifically, fragment attractiveness had a significantly larger influence on predicted likings for familiar fragments $\left(\beta=5.95, t(2452)=11.88, p<.0001, R^{2}=\right.$ .46) than for unfamiliar ones $\left(\beta=4.04, t(1974)=8.96, p<.0001, R^{2}=.37\right), F(1,4426)=7.13, p$ $<.01$. This moderating effect of fragment familiarity was not observed for evaluations of the whole $(p>.2)$.

Similarly, fragment familiarity significantly increased discrepancy between predictions and evaluations of the whole products $(M=8.03$ vs. 3.31$), F(1,84)=9.84, p<.01$. That is, compared to their actual evaluation of the whole, respondents held significantly more optimistic 
expectations about the product for familiar fragments than for unfamiliar ones. This finding is in support of our prediction.

Response Times. Using log-transformed response time data, we replicated the differential effect of fragment valence on response latencies uncovered in Study 1. Attractive fragments were associated with longer response times than unattractive ones $(M=3.41$ vs. 3.01 seconds $), F(1$, $84)=18,65, p<.0001$. This is consistent with the finding that anchoring effects were stronger for attractive than for unattractive fragments.

\section{Discussion}

In Study 2 the task setting differed from that in the previous study in two notable ways: participants were provided with less visualization guidance when making forecasts, and different affective response scale were used to reduce the ease of recall of fragment ratings at the time forecasts were made. Despite these differences, we replicated the key findings of the earlier study. The more important contribution of the study, however, was that it provided more direct support for the hypothesis that extrapolation judgments were made through an anchoring-andadjustment mechanism by finding that the strength of fragment carry-over effects was moderated by participants' familiarity with the fragment. The more familiar the fragment, the greater difficulty participants seemed to have looking beyond their immediate affective responses to the fragment to imagine how they would respond to it in a new context (here, in a shirt pattern).

An obvious limitation of the study, however, is that fragment familiarity is but one of a number of factors that are predicted to moderate anchoring effects both across consumers and within consumers over time. As noted earlier, one source of individual variation may be visualization skills: consumers with greater visualization skills are better able to picture completed images in their mind and thus should be much less susceptible to the biasing effect of 
initial fragments. Likewise, one might expect extrapolation processes to vary over time for consumers due to both situational changes and learning. Specifically, if visualization is hindered by cognitive load as we theorized, we should expect the strength of the anchoring bias to vary depending on the ability of the consumer to narrowly focus on the task: a consumer might form accurate anticipatory judgments of the appeal of a room color when contemplated in a quiet show room, but poor judgments when the choice is made under time pressure in a busy Home Depot. Finally, there may also be a long-term learning effect: the more experience consumers have in performing mental extrapolation tasks and observing their own errors, the less their judgments will be prone to anchoring biases. These predictions were tested in study 3 .

Study 3

\section{Method}

Participants. One hundred and twenty-six college students were recruited in a wall paper prediction task similar to that described in Study 1. The task was part of a 40-minute long experimental session that included other unrelated studies. Each participant received $\$ 10$ for their participation. Six participants did not complete all parts of the study and their responses were excluded from the analysis.

Procedure. The study consisted of three phases. Participants first went through a short training session (see details in the Design section below), followed by the prediction task and measures of processing style. In the prediction task, participants were first shown a series of four wall paper fragments that varied in attractiveness as determined by results from study 1 . For each fragment, participants were asked to indicate how attractive they anticipated a wall paper to be with that fragment on a 15-point scale ( $1=$ "Very Ugly" and $15=$ "Very Attractive"). After a filler task, they were shown on the computer screen the wall papers constructed with each of the 
fragments and were asked to evaluate the wall papers on the same 15-point scale. The same set of dependent measures as in study 1 were collected. Finally, participants completed the style of processing (SOP) questionnaire that measured their preference for and hence skills in processing visual and verbal stimuli. The style of processing questionnaire was taken from Childers, Houston, and Heckler (1985), which has been shown to have good reliability and validity. The questionnaire was labeled as a separate study that was not related to the previous tasks.

Design. The study included three between-subjects factors: training, cognitive load, and visualization skills. During the training session, those in the training condition made predictions for five fragments and then evaluated the whole wall paper immediately after making each prediction. Those in the control (or no training) condition only evaluated the whole wall papers without viewing their respective fragments. Cognitive load was imposed for half of participants during the prediction task. The procedure was as follows: before showing each fragment, the screen first showed a five-letter string (all in uppercase) for four seconds. The on-screen instruction asked participants to memorize the letter string as accurately as possible and also told them that they would be asked to recall the letter string after each prediction. A random letter string was selected for each fragment. After the letter string disappeared from the screen, the fragment with the prediction question would appear on the screen. Once a participant had made his or her prediction, the screen would ask the participant to key in the letter string that had appeared on the screen prior to the fragment. The other half of participants were not shown the letter strings. Processing style was measured through the style of processing scale. The scale consists of two subscales: visual subscale and verbal subscale, each with eleven statements. Participants indicated the degree to which each statement described themselves on a 4-point Likert scale ( $1=$ "Always true" and $4=$ "Always false"). Visual and verbal scores were 
calculated for each participant by summing up the eleven items for each subscale. Some items were reverse coded so that higher scores on each subscale indicate greater preference for each processing style. In addition, fragment attractiveness was manipulated within-subjects as each participant viewed two attractive and two unattractive fragments. Fragments were selected from those used in study 1 and the manipulation of attractiveness was based on participants' ratings in study $(M=8.7$ vs. 4.5 for attractive and unattractive fragments, respectively), $F(1,43)=78.80, p$ $<.0001$. Figure 2 (lower panel) shows the fragments used in study 3 .

\section{Results}

Preliminaries. Overall, participants in the cognitive load condition correctly recalled $71 \%$ of the letter strings, indicating that the cognitive load manipulation was not ignored by participants. Recall accuracy did not affect participants' performance on the extrapolation task. The style of processing measures also achieved satisfactory reliability, Cronbach $\alpha=.77$ and .81 for visual and verbal subscales, respectively. Moreover, the two subscales were uncorrelated, $r=$ $.10, p>.2$. Since verbal scores did not have any significant effect on any of the dependent measures, it was not included in the analyses reported below. Participants were median split into two groups with high and low visual scores $(M=35.4$ vs. 29.0$), t(116)=14.26, p<.0001$, who will be henceforward referred to as visualizers and non-visualizers. The short training exercise had no significant effect $(p s>.3)$ on any of the dependent measures, an issue we will return to in the discussion. Thus, responses were collapsed across the training conditions for the analyses reported below. Adding the variable for training condition did not affect the results reported here. Table 3 provides the mean responses by treatment conditions. 
As expected, evaluations of the wholes revealed only a main effect of fragment attractiveness $(M=8.37$ and 6.45 for attractive and unattractive fragments, respectively), $F(1$, $116)=55.14, p<.0001$. Cognitive load and visualization skills did not affect the whole evaluations. Similarly, predicted likings were significantly influenced by fragment attractiveness, $F(1,116)=421.04, p<.0001$. Congruence between predictions and evaluations of the whole products (i.e., predicted liking - actual evaluation of the whole) showed a significant effect of fragment attractiveness $(M=1.76$ and -.74 for attractive and unattractive fragments, respectively), $F(1,116)=231.20, p<.0001$, replicating the results from earlier studies.

Moderating Role of Cognitive Load. Cognitive load did not influence evaluations of the wholes $(p>.3)$ but the data revealed a significant fragment attractiveness $\mathrm{x}$ cognitive load interaction in predicted likings, $F(1,116)=4.02, p<.05$. Specifically, fragment attractiveness had a stronger influence on predicted likings when the decision maker was under cognitive load $(M=10.45$ vs. 5.46 for attractive and unattractive fragments $)$ than in the no-load condition $(M=$ 9.80 vs. 5.97).

A similar pattern emerged from the data on the congruence between predicted likings and actual evaluations of the whole products, $F(1,116)=5.05, p<.05$. The discrepancy was stronger in the cognitive load condition ( $M=1.97$ vs. -.87 for attractive and unattractive fragments) than in the no-load condition $(M=1.57$ vs. -.68).

Moderating Role of Visualization Skills. The data also revealed a marginally significant fragment attractiveness $\mathrm{x}$ visualization skills interaction in predicted likings, $F(1,116)=3.76, p$ $<.10$. Specifically, the influence of fragment attractiveness on predicted likings was more muted for visualizers ( $M=9.98$ vs. 5.81 for attractive and unattractive fragments $)$ than for non- 
visualizers ( $M=10.33$ vs. 5.57). Likewise, visualizers also displayed higher congruence between predicted likings and actual evaluations of the whole products $(M=1.44$ vs. -.72 for attractive and unattractive fragments) than did non-visualizers $(M=2.16$ vs. -.91$)$. The visualization skill $\mathrm{x}$ fragment attractiveness interaction was significant, $F(1,116)=5.47, p<.05$. These results confirmed the hypothesis that skills at visualization helped reduce the decision bias in the present task. They also suggest that the experts among our participants followed rather similar decision strategies as their more novice counterparts.

Response Times. Mirroring previous results, participants on average spent more time on attractive fragments than on unattractive ones in the absence of cognitive load $(M=3.80 \mathrm{vs.} 3.16$ seconds), $F(1,116)=11.12, p<.001$. As may be expected, cognitive load significantly increased the response time for the prediction task $(M=4.42$ and 3.48 seconds $), F(1,116)=13.84, p<$ .0001 . In contrast, we found no effect on response time of processing style $(M=3.91$ vs. 3.89 seconds for visualizers and non-visualizers, $p>.9$ ). One interpretation of this latter finding is that it suggests that the greater accuracy achieved by participants with greater visualization skills accrued to more efficient—rather than simply more-cognitive elaboration.

\section{Discussion}

Study 3 examined the effect of three factors that were hypothesized to influence the accuracy of mental extrapolations and the use of anchoring strategy: training (or experience) in mental extrapolation judgments, cognitive load during predictions, and visualization skills. We predicted that training and preference for processing visual stimuli would lead to superior performance at the mental extrapolation task whereas cognitive load would have the opposite effect. The results were largely consistent with these expectations. Cognitive load had a 
significant detrimental effect on judgment accuracy while increasing the decision time, and visualizers exhibited smaller errors in the task than non-visualizers.

Task experience, however, did not produce the expected beneficial effect for our participants. Two factors may have contributed to the null effect. First, the training exercise may be too short and insufficient (each participant had only five trials), especially given that our participants were likely to have had little prior experience in wall paper purchases. Expertise is typically a product of extensive experience and thinking; thus the limited experience provided in the training exercise may not be enough to make an appreciable increase in our participants' expertise. Secondly, the limited learning that participants may have gathered from the training exercise may be specific to the training stimuli, instead of any general theory about how the visual appeal of a fragment relate to the overall appeal of a wall paper. For example, after viewing how a pattern of bubbles was transformed into a wall paper, a consumer may take away the lesson about how to extrapolate a bubbles pattern in the future, instead of a rule of thumb about how to extrapolate patterns in general. Decision makers tend to treat each decision as a unique case, which inhibits effective learning and development of true expertise in the extrapolation task (Tversky \& Kaheman, 1986). Thus, the issue of how consumers may develop expertise in these extrapolation judgments remains unresolved.

\section{General Discussion}

This paper took a first step toward understanding the psychological basis of a common, but under-researched, form of consumer inference: predicting the likely appeal of whole product images from small sample fragments. Three studies provided evidence that such judgments are often marked by an anchoring bias in which predicted evaluations of wholes are excessively influenced by the localized appeal of fragments. As a result, there is a tendency to make overly 
optimistic predictions about the appeal of wholes made from attractive fragments and overly pessimistic predictions about the appeal of wholes made from unattractive fragments. We find, however, that this bias is not necessarily a universal: its strength is moderated by situational and interpersonal factors, such as the perceived familiarity of the fragment, the cognitive load that accompanies the extrapolation task, and visualization skills of the decision maker.

These findings hold several implications for marketers whose businesses require consumers to engage in such extrapolation tasks such as home improvement stores and apparel catalogs. First, the prevalence of forecast errors substantiates the common intuition that mental extrapolation is not something we naturally do well. How can mental extrapolation judgments be improved? The simplest, of course, is to provide visualization aids, such as those we described at the outset (e.g., the tools at the Dutch Boy website). But even if such aids are infeasible the current work suggests that predictive accuracy might be improved by designing retail environments that are more conducive to visualization. For example, retailers could reduce the detrimental effects of cognitive load simply by reducing the number of fragments being considered by consumers and encouraging them to take samples with them outside the store. Likewise, it might be possible for retailers to encourage more accurate visualization by warning consumers against making typical projection errors, such as basing choices on the local appeal of a fragment.

While the current study provides initial insights into how consumers make mental extrapolation judgments, it is important to emphasize that the data come from a task setting that simplified many of the complex features that arise in real world extrapolation judgments. For example, one aspect of visual extrapolation judgments that arises in the real world that we controlled for here was the contaminating effect of the context in which a fragment is first 
viewed. When consumers buy furniture, for example, the challenge they face is not only imagining what a piece of furniture would look like in one's home, but also parsing out the potential contaminating influence of the appeal of the showroom in which the piece is first viewed. The effect of such initial context, however, could cut two ways. On one hand, viewing a piece in an attractive model living room might be hypothesized to amplify optimism biases since the holistic attractiveness of the room may carry over to independent impressions of the piece. On the other hand, the presence of a living room context may aid consumers' attempts to visualize the piece in their own homes. Thus, one interest topic for future research might thus is to examine the flip-side of the problem we considered here, namely, the ability of consumers to form accurate impressions of the independent appeal of fragments upon seeing them in completed wholes.

Likewise, it would be interesting to investigate the degree to which the findings uncovered here generalize to other extrapolation tasks, such as judgments about the likely attractiveness of movies from trailers, software from limited-capability demonstration versions, and food items from taste samples. What makes such tasks interesting is that they require consumers to form more complex, dynamic, models of wholes than that which was required here (e.g., imagining how enjoyable the elements shown in the trailer would be extended to 120 minutes in a whole film). While we suspect that such judgments will be again be prone to anchoring biases (e.g., entertaining trailers would give rise to overly optimistic forecasts of film quality), predictions of dynamic wholes might also be influenced by psychological factors that likely played only a minor role here (such as whether the trailer evokes or suppresses feelings of curiosity). 


\section{References}

Brickman, P., Coates, T., \& Janoff-Bulman, R. (1978). Lottery winners and accident victims: Is happiness relative? Journal of Personality and Social Psychology, 36, 917-927.

Chapman, G. B., \& Johnson, E. J. (1999). Anchoring, activation, and the construction of values. Organizational Behavior and Human Decision Processes, 79, 115-153.

Childers, T. L., Houston, M. J., \& Heckler, S E. (1985). Measurement of individual differences in visual versus verbal information processing. Journal of Consumer Research, 12, 125134.

Dahl, D. W., Chattopadhyay, A., \& Gorn, G. J. (1999). The use of visual mental imagery in new product design. Journal of Marketing Research, 36. 18-28.

Fiske, S. T. (1980). Attention and weight in person perception: the impact of negative and extreme behavior. Journal of Personality and Social Psychology, 38, 889-906.

Förster, J. (2004). How body feedback influences consumers' evaluation of products. Journal of Consumer Psychology, 14, 416-426.

Gehring, W. J., \& Willoughby, A. R. (2002). The medial frontal cortex and the rapid processing of monetary gains and losses. Science, 295, 2279-2282.

Gilbert, D. T., Gill, M. J., \& Wilson, T. D. (2002). The future is now: Temporal correction in affective forecasting. Organizational Behavior and Human Decision Processes, 88, 43044.

Kahneman, D., \& Schkade, D.A. (1998). Does living in California make people happy? A focusing illusion in judgments of life satisfaction. Psychological Science, 9, 340-6.

Kardes, F. R., Posavac, S. S., \& Cronley, M. L. (2004). Consumer inference: A review of processes, bases, and judgment contexts. Journal of Consumer Psychology, 14, 230-56. 
Keller, A. P., \& Block, L. G. (1997). Vividness effects: A resource matching perspective. Journal of Consumer Research, 24, 294-305.

Kosslyn, S. M. (1994). Image and brain: The resolution of the imagery debate. Cambridge, MA: MIT Press.

Loewenstein, G. (1996). Out of control: Visceral influences on behavior. Organizational Behavior and Human Decision Processes, 65, 272-92.

Loewenstein, G., O’Donoghue, T., \& Rabin, M. (2003). Projection bias in predicting future utility. Quarterly Journal of Economics, 118, 1209-48.

Loewenstein, G., \& Schkade, D. (1999). Wouldn't it be nice? Predicting future feelings, in D. Kahneman, E. Diener, \& N. Schwarz (Eds.). Well-being: The foundations of hedonic psychology (pp. 85-105). New York: Russell Sage Foundation.

MacInnis, D. J., \& Price, L. L. (1987). The role of imagery in information processing: Review and extensions. Journal of Consumer Research, 13, 473-91.

Meyer, R. J. (1981). A model of multiattribute judgments under attribute uncertainty and informational constraint. Journal of Marketing Research, 18, 428-41.

Mussweiler, T., \& Strack, F. (1999). Hypothesis-consistent testing and semantic priming in the anchoring paradigm: A selective accessibility model. Journal of Experimental Social Psychology, 35, 136-164.

Shepard, R. N., \& Cooper, L. A. (1986). Mental images and their transformations. Cambridge, MA: The MIT Press.

Simmons, C. J. \& Lynch, J. G., Jr. (1991). Inference effects without inference making? Effects of missing information on discounting and use of presented information. Journal of Consumer Research, 17, 477-91. 
Tversky, A., \& Kahneman, D. (1974). Judgment under uncertainty: Heuristics and biases, Science, 185, 1124-31.

Tversky, A., \& Kahneman, D. (1986). Rational choice and the framing of decisions. Journal of Business, 59, S251-278.

Ubel, P. A., Loewenstein, G. \& Jepson, C. (2005). Disability and sunshine: Can predictions be improved by drawing attention to focusing illusions or emotional adaptation? Journal of Experimental Psychology: Applied, 11, 111-123.

Wilson, T. D., Houston, C. E., Etling, K. M., \& Brekke, N. (1996). A new look at anchoring effects: Basic anchoring and its antecedents. Journal of Experimental Psychology: General, 125, 387-402.

Wilson, T. D., \& Gilbert, D. T. (2003). Affective forecasting, in M. Zanna (Ed.), Advances in Experimental Social Psychology, 35 (pp. 345-411), New York: Elsevier.

Wilson, T. D., Wheatley, T., Meyers, J. M., Gilbert, D. T., \& Axsom, D. (2000). Focalism: A source of durability bias in affective forecasting. Journal of Personality and Social Psychology, 78, 821-36.

Zajonc, R. B. (1980). Feeling and thinking: Preferences needs no inferences. American Psychologist, 35, 151-175. 
Table 1

Mean Evaluations in Study 1

\begin{tabular}{lcccc}
\hline Fragment Type & $\begin{array}{c}\text { A. Fragment } \\
\text { Evaluation* }\end{array}$ & $\begin{array}{c}\text { B. Predicted } \\
\text { Liking of the } \\
\text { Whole* }\end{array}$ & $\begin{array}{c}\text { C. Experienced } \\
\text { Liking of the } \\
\text { Whole* }\end{array}$ & $\begin{array}{c}\text { D. Discrepancy } \\
\text { (B - C) }\end{array}$ \\
\hline All & 7.08 & 6.34 & 6.16 & 0.18 \\
$(\mathrm{~N}=792)$ & $(3.83)$ & $(3.87)$ & $(3.81)$ & $(3.61)$ \\
Unattractive & 4.27 & 3.82 & 4.73 & -0.91 \\
$(\mathrm{~N}=385)$ & $(2.60)$ & $(2.75)$ & $(3.31)$ & $(3.14)$ \\
Attractive & 9.73 & 8.71 & 7.51 & 1.20 \\
$(\mathrm{~N}=407)$ & $(2.75)$ & $(3.22)$ & $(3.77)$ & $(3.72)$ \\
\hline
\end{tabular}

Note. Standard deviations are in parentheses. $\mathrm{N}=$ \# of observations.

*Measured on 15-point scale, $1=$ Not attractive at all, and $15=$ Extremely attractive 
Table 2

Mean fragment ratings and responses in study $2^{a}$

\begin{tabular}{|c|c|c|c|c|c|}
\hline & & & & C. Actual & D. \\
\hline Fragment & Fragment & A. Fragment & B. Predicted & Liking of & Discrepancy \\
\hline Attractiveness & Familiarity & Rating $^{\mathrm{b}}$ & Liking $^{c}$ & Whole $^{c}$ & $(B-C)$ \\
\hline \multirow[t]{4}{*}{ Attractive } & Unfamiliar & 2.04 & 44.84 & 39.30 & 6.12 \\
\hline & $(\mathrm{N}=713)$ & $(0.97)$ & $(28.86)$ & $(30.81)$ & $(26.96)$ \\
\hline & Familiar & 2.03 & 44.75 & 34.60 & 10.33 \\
\hline & $(\mathrm{N}=1435)$ & $(0.95)$ & $(25.72)$ & $(28.22)$ & $(25.61)$ \\
\hline \multirow[t]{4}{*}{ Unattractive } & Unfamiliar & -1.93 & 21.09 & 19.38 & 1.78 \\
\hline & $(\mathrm{N}=1331)$ & $(0.82)$ & $(23.54)$ & $(23.07)$ & $(23.42)$ \\
\hline & Familiar & -1.81 & 18.95 & 14.07 & 5.09 \\
\hline & $(\mathrm{N}=1106)$ & $(0.80)$ & $(21.42)$ & (19.06) & $(22.23)$ \\
\hline
\end{tabular}

Notes:

${ }^{a}$ Standard deviations are in parentheses. $\mathrm{N}=$ \# of observations.

${ }^{\mathrm{b}}$ Measured on a 7-point scale $(-3$ to +3$)$

${ }^{\mathrm{c}}$ Measured on a 101-point scale $(0-100)$ 
Table 3

Mean responses by treatment conditions in study 3

\begin{tabular}{|c|c|c|c|c|c|}
\hline $\begin{array}{l}\text { Processing } \\
\text { Style }\end{array}$ & $\begin{array}{l}\text { Cognitive } \\
\text { Load }\end{array}$ & Fragment & $\begin{array}{l}\text { A. Predicted } \\
\text { Liking }\end{array}$ & $\begin{array}{c}\text { B. Actual } \\
\text { Liking of } \\
\text { Whole }\end{array}$ & $\begin{array}{c}\text { C. } \\
\text { Discrepancy } \\
(A-B)\end{array}$ \\
\hline \multirow[t]{8}{*}{$\begin{array}{l}\text { Non- } \\
\text { Visualizers }\end{array}$} & No Load & Unattractive & 6.18 & 6.82 & -0.64 \\
\hline & $(\mathrm{N}=52)$ & & $(2.31)$ & (3.19) & $(2.69)$ \\
\hline & & Attractive & 9.94 & 7.94 & 2.00 \\
\hline & & & $(3.00)$ & $(3.24)$ & $(3.60)$ \\
\hline & Load & Unattractive & 4.98 & 6.14 & -1.16 \\
\hline & $(\mathrm{N}=52)$ & & $(2.36)$ & $(3.18)$ & $(2.14)$ \\
\hline & & Attractive & 10.72 & 8.40 & 2.32 \\
\hline & & & $(2.43)$ & $(3.68)$ & (3.98) \\
\hline \multirow[t]{8}{*}{ Visualizers } & No Load & Unattractive & 5.79 & 6.30 & -0.52 \\
\hline & $(\mathrm{N}=56)$ & & $(2.80)$ & $(3.36)$ & $(2.96)$ \\
\hline & & Attractive & 9.68 & 8.54 & 1.14 \\
\hline & & & $(3.20)$ & $(3.57)$ & $(3.25)$ \\
\hline & Load & Unattractive & 5.84 & 6.53 & -0.69 \\
\hline & $(\mathrm{N}=60)$ & & $(2.74)$ & $(3.76)$ & $(2.22)$ \\
\hline & & Attractive & 10.23 & 8.55 & 1.69 \\
\hline & & & $(2.88)$ & $(3.75)$ & $(3.77)$ \\
\hline
\end{tabular}

Note. Standard deviations are in parentheses. $\mathrm{N}=\#$ of participants. 


\section{Figure Captions}

Figure 1: Example of fragment (left) and referent wall paper image (right) with the associated response scale and sample fragments (lower panel) in study 1

Figure 2: Screenshot for the prediction task and sample stimuli in study 2 
Figure 1: Example of fragment (left) and referent wall paper image (right) with the associated response scale and sample fragments (lower panel) in study 1

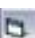

Pleose examine the following pottern

Imagine the pattern below is a tile. If you replace the tiles in the configuration on the right with this pattern in the same manner to form a wallpaper. Of course, the wallpaper will consist of many more tiles. IMAGINE $A$ WALLPAPER MADE WITH THIS PATTERN.

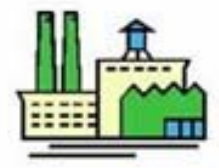

This configuration consists of four IDENTICAL tiles.

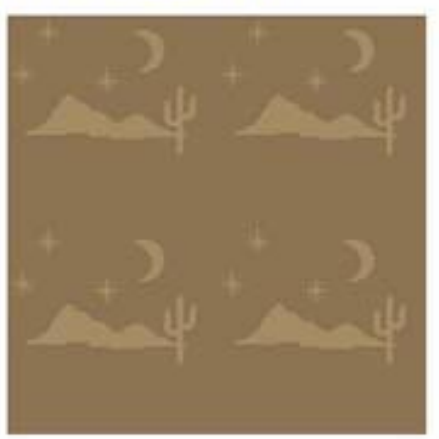

To your best guess, how attractive will a wallpoper be if the pattern on the left is arranged in the same manner os in the configuration on the right?

$\begin{array}{rrrrrrrrrrrrrrrrrrrrrr}\text { Very Ugly } & C & C & C & C & C & C & C & C & C & C & C & C & C & C & C & \text { Very } \\ -7 & -6 & -5 & -4 & -3 & -2 & -1 & 0 & +1 & +2 & +3 & +4 & +5 & +6 & +7 & \text { Attractive }\end{array}$
Sample Attractive Fragments
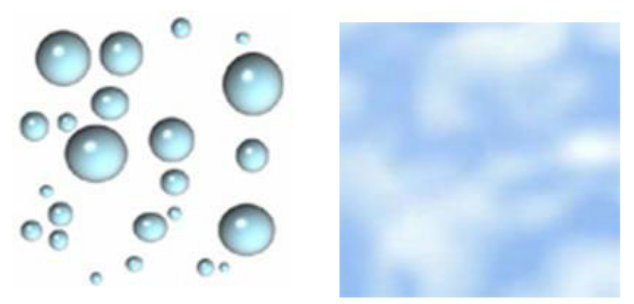

Sample Unattractive Fragments
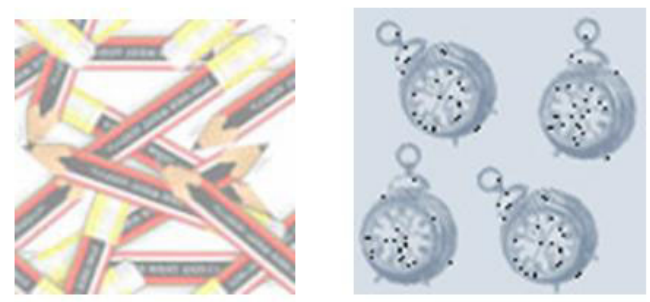
Figure 2: Screenshot for the prediction task and sample stimuli in study 2

\section{Catalog}

A designer pattern for the shirts is shown below along with a sample shirt outline.

Note: The partern is shown here about 4 times larger than the actual

size on the scale of the sample shirt for a more detailed view. The patterns will appear on the shirt IIU A TILED FASHIOH.

Please indicate on the scroll bar below how attractive or ugly you think the shirt will appear.
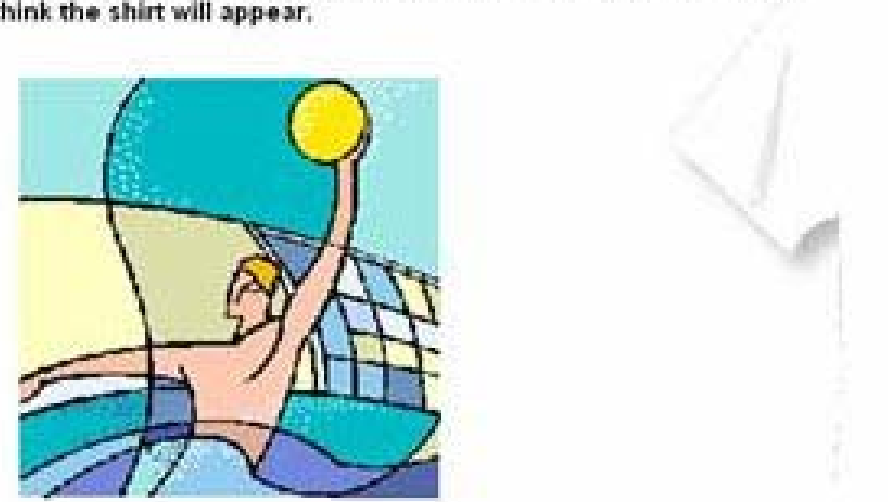

When the above pattern is applied to the shirt. how attractive or ugly do you think the shirt will look? (Please note that vou have to move the scroll bar to continue)

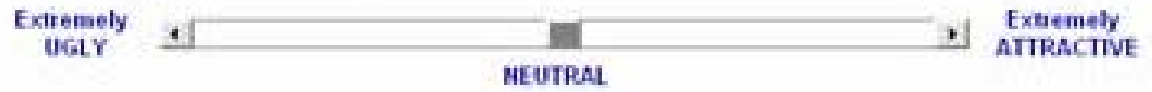

Cick tere to continue:

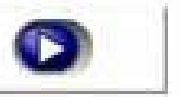

Sample Stimuli
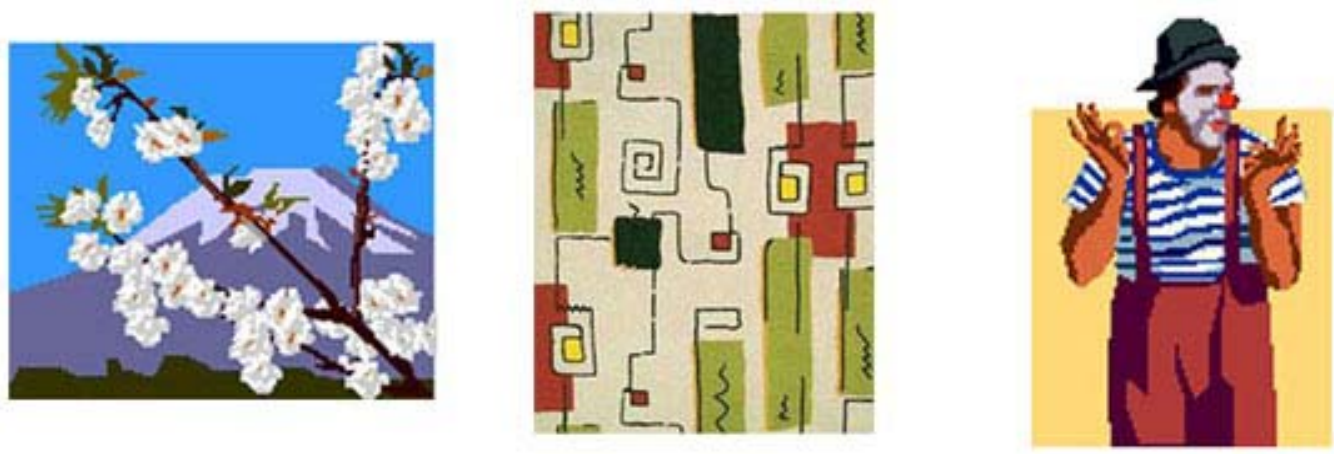